\title{
Implementing a Non-Invasive Brain Temperature Monitoring System with Two-Type RF Switches Antenna
}

\author{
Syed Jamalullah. R and L. Mary Gladence \\ Sathyabama Institute of Science and Technology, Chennai, India
}

\section{ABSTRACT}

The research offers an extensive cavity-based antenna for the active monitoring via sensations microwave radiometry of brain temperature. The antenna will be $20 \mathrm{~mm}$ in diameter and will measure $4.56 \mathrm{~mm}$ in height from 2.723 to 5.032 $\mathrm{GHz}$. The layout of the antenna is a three-slotted, hexagonal patch similar to the picture of the human head. The analyses of the Specific Absorption Rate (SAR) show that the antenna can access the deep brain's microwaving energy that is suitable for the brain temperature monitoring technique as assistance. The antenna is then attached to a two-type switch system for strong ESD (Electromechanical and Solid-State RF Switches) immunity and high replacement capacity.

KEY WORDS: MICROWAVE RADIOMETRY-BRAIN TEMPERATURE-SPECIFIC ABSORPTION RATE (SAR) - RF SWITCHES.

\section{INTRODUCTION}

The constant monitoring of the deep brain temperature is one of the most important diagnostic markers for mental health. The brain relief in preterm infants, hyperthermia analyses and hypothermic problems during operations, cancer cure thermotherapy and newborn treatment are among the situations needed for such analyses. Effective brain temperature monitoring is impeded by problems associated with deep brain access. Because of its overcontact character, replacement microwave radiometry (MWR) techniques can also be deployed. The MRI and MRS methods have been two comparable approaches, but their wider use was prevented due of their excessive cost and limits of intuitive measures. In hospitals, they have been widely used. MRI and MRS were both comparable techniques. This pertains also to positron emission tomography (PET) and infrared functional spectroscopy (FNIR). It is thus greatly praised that the brain temperature is inexpensive and convenient for use at the treatment

Biosc Biotech Res Comm P-ISSN: 0974-6455 E-ISSN: 2321-4007



Identifiers and Pagination

Year: 2021 Vol: 14 No (5) Special Issue

Pages: 245-247

This is an open access article under Creative

Commons License Attribn 4.0 Intl (CC-BY). DOI: $h t t p: / / d x$.doi.org/10.21786/bbrc/14.5/44 facility, including passive and noninvasive microwave radiometric monitoring.

Deep measurement of the brain temperature presents several problems owing to extraordinarily low microwave energy radiation between $10-14 \mathrm{~W} / \mathrm{MHz}$ at $37^{\circ} \mathrm{C}$. This energy is equivalent to $-80 \mathrm{~dB} \mathrm{~m}$ at a bandwidth of $1 \mathrm{GHz}$, which requires narrow band head antennas to assemble the maximum of power. In addition, fragile and heavy-gain RF amplifiers have been needed to achieve appropriate attenuation. Set up around the head, as indicated in Figure 1, assorted head-matched antennas with a greater hyperactivity of flexible, dielectric surface, as shown in Fig. 1(a). The range may also be radially moved over the head to various points for any sub-set of head measurement.

Figure 1(b) provides a block layout of a proposed instance for measuring brain temperature. The system consists of two RF-type (Electromechanical and Solid State) switches intended for single or multiple antennas to be connected to the RF-front. The outcome will be the ideal solution in two phases by connecting two-type switches. The received RF signals are transcribed and delivered to a digital signal processor which modifies the temperature measuring panel qualitatively and geographically. This article depicts a wideband antenna with a matching head with a compact design and a unique loop design.
Article Information

Received: $15^{\text {th }}$ Jan 2021 Accepted after revision: $27^{\text {th }}$ Mar 2021 
Figure 1: Brain Temprature Monitoring System (a) Antenna placement (b) Block Diagram



Table 1. Parametric Result for RF Switches

\begin{tabular}{|l|c|c|}
\hline Parameters & Electromechanical & Solid State \\
\hline Frequency range & from [DC] & from kHz \\
\hline Repeatability & Good & Excellent \\
\hline Isolation & Excellent & Good \\
\hline ESD immunity & High & Low \\
\hline
\end{tabular}

II. Overview of RF Switches: An RF switch is a tool for setting higher frequency signals using propagation paths. RF switches are often utilized to route signals between instruments and Device under test (DUT) under this automation system. By incorporating a switch to a switch module system you may transmit signals from different devices to single or many DUTs. It allows tests using the same framework and reduces the requirement for frequent contact and disruptions of service. This might simplify the huge screening procedure and increase network performance in cases of heavy output.

The two main RF switch types provide expertise:

(i) Electromechanical RF Switch: The simple electromagnetic induction principle underpins electromechanical switches. The operation of switching is mechanical. Electromechanical switches blend physically moving interaction measurements with a circuit shutdown switch to re-assign messages from one part to the next. These switches are always constructed using wavelength interconnections to incorporate more power. Not every electromechanical switch is equal. It makes sense. They cannot even be designed to accommodate heavy-power hot-switching transmissions. An electric electromechanical switch, for example, spins an electrical connection between numerous switch sites.

(ii) Solid State RF Switch: The solid-state relay and the SSR do not have mechanical parts but rather incorporate the electric and optic properties of solidstate semiconductors to process its access to detached performances or toggle features in electromechanical relays (EMRs), using loops, magnetic fields, springs and mechanical interactions to activate and toggle an outcome. SSR includes total isolation surrounding their input and output interactions as a traditional electromechanical relay, as it perform with a very high near-finitely rough friction (open) but extremely low friction while conducting (closed).

III. Proposed System: The antenna was placed under the fantasy of the human head in an extended posture. The fantasy includes skin, bone, brain, muscle and countless more biological cells provided by CST Microwave Studio. The default result deviation reveals the antenna has 2.723-5.032 GHz capacity range (2,309 GHz). This largescale reaction fulfils the body's implementation criteria. Their specific rate of absorption (SAR) in their operation range has been evaluated at two frequencies in order to determine the dependability of the antenna for deep brain temperature test. The suggested specific absorption rate (SAR) is the verifying energy collection of the deep brain antenna for 3.5 and $4.5 \mathrm{GHz}$. This may be deduced that the skull (bone) has negligible failure and the bulk of energy disappears into the brain. This is the influence on a balanced model of brain fantasy. The parameters are reasonably optimal with its positioning system by introducing a two-type switching mechanism into the suggested antenna. The multi-type switching system is shown as a feature table. The above figure shows that when the two switches are applied in the non-invasive antenna, the results of the parameters are excellent.

\section{CONCLUSION}

A broad band head matched antenna for deep brain temperature control is identified in this document. A large transmission range of $2.723-5.032 \mathrm{GHz}$ appears to be on this idea antenna. The examination of the Specific Absorption Rate (SAR) demonstrates that deep brain energy may be gathered and then utilized as an average brain temperature. This antenna is suitable for brain temperature monitoring systems thanks to its small size and outstanding human head fitting.

\section{REFERENCES}

Dario B. Rodrigues, "Development and optimisation of a UltraWide Band and Portable Microwave Antenna for Brain Temperature Radiometric Monitoring", IEEE Transactions on Biomedical Engineering, vol. 61, no. 7, july 2014.

Dittmar, K. Maruyma, "A noninvasive wearable sensor for the measurement of brain temperature", Proceedings of the 28th IEEE EMBS Annual International Conference, New York City, USA, Aug 30-Sept 3, 2006, "Feasibility of Noninvasive Measurement of Deep Brain Temperature in Newborn Infants by Multi frequency Microwave Radiometry”, IEEE Transactions on Microwave Theory and Techniques, Vol. 48, No. 11, November 2000.

G.M.J. Van Leeuwen, "Brain Temperature Monitoring Algorithm Using Microwave Brightness Temperatures," Electrornic Letters, vol. 37, 6th March, 2001.

Louis Atallah ,IEEE Journal of Biomedical and Health Informatics, "Unobtrusive Monitoring of Neonatal Brain 
Temperature Using a Zero-Heat-Flux Sensor Matrix," Vol. No. 20, January 1, 2016

Paul R. Stauffer, Irene S. Karanasiou, "Non-Invasive Microwave Radiometry Brain Temperature Measurement: Head Phantom and Clinical Case demonstration," Neuroradiology Journal, vol. 27, pp. 3, 2014. "Study of a Brain Hyperthermia System providing also Passive
Brain Temperature Monitoring”, Proceedings of the 28th IEEE EMBS Annual International Conference New York City, USA, Aug 30-Sept 3, 2006.

S. Gabriel, R. W. Lau, and C. Gabriel, "Biological Tissue Dielectric Properties: III. Parametric models for a dielectric tissue spectrum," Phys. Med. Biol., vol. 41, no. 11, November 1996. 